\title{
Relationship between miR-155 and miR-146a polymorphisms and susceptibility to multiple sclerosis in an Egyptian cohort
}

\author{
MARWA AHMED ALI ${ }^{1}$, OLFAT GAMIL SHAKER ${ }^{2}$, HANAA MOHAMED EID ${ }^{3}$, EMAN ELSAYED MAHMOUD ${ }^{4}$, \\ EMAN MAHMOUD EZZAT ${ }^{5}$ and SYLVANA NADY GABER ${ }^{3}$ \\ ${ }^{1}$ Department of Medical Biochemistry and Molecular Biology, Faculty of Medicine, Fayoum University, Fayoum 63514; \\ ${ }^{2}$ Department of Medical Biochemistry and Molecular Biology, Faculty of Medicine, Cairo University, Cairo 11562; \\ Departments of ${ }^{3}$ Medical Microbiology and Immunology, ${ }^{4}$ Clinical and Chemical Pathology and \\ ${ }^{5}$ Internal Medicine, Faculty of Medicine, Fayoum University, Fayoum 63514, Egypt
}

Received July 15, 2019; Accepted December 13, 2019

DOI: $10.3892 /$ br.2020.1286

\begin{abstract}
Multiple sclerosis (MS) is an autoimmune disorder of the central nervous system. It was previously demonstrated that miR-155 and miR-146a served a vital role in the pathophysiology of MS, and single nucleotide polymorphisms in miR-155 and miR-146a were found to be associated with the susceptibility to different autoimmune diseases, such as systemic lupus erythematosus, rheumatoid arthritis and type I diabetes. The aim of the present study was to analyze the association between susceptibility to MS and two genetic polymorphisms (miR-155 rs767649 A>T and miR-146a rs57095329 $A>G$ ) in a cohort of Egyptian patients. The presence of the two polymorphisms were analyzed in 114 patients with MS and 152 healthy controls using quantitative PCR. The present study demonstrated for the first time that: The TT genotype and $\mathrm{T}$ allele in miR-155 (rs767649 $\mathrm{A}>\mathrm{T}$ ) polymorphism were associated with an increased risk of MS; the miR-146a (rs57095329 A>G) mutated $\mathrm{G}$ allele conferred protection against the development of MS in all genetic models; miR-155 rs767649 A>T was a risk associated polymorphism of MS in females, but not in males; and miR-155 rs767649 AT/TT and miR-146a rs57095329 GG genotypes showed significantly higher distributions among patients with higher Expanded Disability Status Scale scores and secondary progressive MS subgroups. Therefore, miR-155 rs767649 polymorphism may confer susceptibility to MS, whereas miR-146a rs57095329 may be protective against MS in an Egyptian cohort.
\end{abstract}

Correspondence to: Dr Marwa Ahmed Ali, Department of Medical Biochemistry and Molecular Biology, Faculty of Medicine, Fayoum University, 32 Zaid Bin Haritha Street, Al Mashtal, Fayoum 63514, Egypt

E-mail: mag04@Fayoum.edu.eg

Key words: multiple sclerosis, miR-155 rs767649, miR-146a rs57095329, polymorphism, quantitative PCR

\section{Introduction}

Multiple sclerosis (MS) is a non-fatal long-lasting autoimmune disease characterized by chronic inflammation and demyelinating neurodegeneration of the central nervous system (CNS) (1). There are three primary clinical subtypes of MS; $\sim 80 \%$ are diagnosed with relapsing-remitting MS (RRMS), with secondary progressive MS (SPMS) developing in the following decades, whereas in a smaller group of patients $(20 \%)$ MS begins with a primary progressive phase (PPMS) (2). The etiology of MS remains unclear; however, several studies have shown that both genetic and environmental factors influence susceptibility to MS (3-5).

MicroRNAs (miRNAs/miRs) constitute a group of 20-22-nucleotide-long non-coding RNAs which regulate target mRNA expression post-transcriptionally through mRNA degradation or translation inhibition (6). miRNAs serve essential roles in the immune system in the development of innate and adaptive immunity and for proper immune function (7). miR-155 regulates the innate and adaptive immune responses in response to infection (8) However, miR-155 dysregulation via overexpression is associated with various inflammatory disorders $(9,10)$. miR-146a is a negative regulator of immune and inflammatory responses (11). miR-155 and miR-146a are associated with the pathogenesis of several autoimmune diseases $(6,12)$, including MS in humans and in experimental autoimmune encephalomyelitis (EAE) lesions in mice, suggesting that miR-155 and miR-146a may be involved in the pathophysiology of MS $(10,13)$.

Single nucleotide polymorphisms (SNPs) are single nucleotide variations that occur at a unique site in the DNA (14). These changes in the DNA sequence can affect the susceptibility to different diseases, including autoimmune diseases (15). SNPs in miR-155 rs767649 and miR-146a rs57095329 are associated with the development of immune-system dysfunctions $(16,17)$. Notably, the selected SNPs (miR-155 rs767649 and miR-146a rs57095329) are located in the regulatory regions of these miRNAs and may thus affect their expression levels (16-21).

Given the regulatory role of miR-155 and miR-146a in the immune response in MS and the possible effect of the miR-155 rs767649 and miR-146a rs57095329 SNPs on their 
respective expression levels, it was hypothesized that these polymorphisms may alter the susceptibility to MS. Therefore, the aim of the present study was to ascertain whether these two polymorphisms are associated with MS susceptibility, and whether these SNPs are associated with demographic and clinical features of MS in an Egyptian cohort. Based on the results of the present study, these two polymorphisms may have value as diagnostic biomarkers for MS and may inform novel new therapeutic approaches for MS treatment.

\section{Materials and methods}

Patient recruitment. The MS cohort of the present study consisted of 114 patients (mean age \pm standard deviation, $31.32 \pm 8.36$ years) who were outpatients or inpatients at the Department of Neurology or Department of Internal Medicine at El Fayoum University Hospital (Fayoum, Egypt), including 96 females and 18 males, with clinically confirmed MS. MS diagnosis was confirmed based on the recommended diagnostic criteria for multiple sclerosis (22). Disease severity was evaluated using the Kurtzke Expanded Disability Status Scale (EDSS) (23). EDSS is a method of measuring disability in patients with MS over time. EDSS scoring depends on an examination by a neurologist. The EDSS score ranges from 0-10 in 0.5-unit increments, with a higher score representing a higher level of disability (23). A value of 6 is a cut-off value, with scores $\geq 6$ meaning a patient who is immobile and requires a walking aid to walk $\sim 100 \mathrm{~m}$ with or without rest $(15,23)$. Patients with concurrent autoimmune or inflammatory diseases or malignancies, patients suspected to be drug or alcohol addicts, or pregnant patients were excluded from the present study.

The 114 patients with MS were classified into two subgroups: Subgroup I consisted of 84 patients with RRMS and subgroup II consisted of 30 patients with SPMS. The medical history of each patient was obtained, including age, age of onset and current treatment. Patients who received MS-specific treatment (immunomodulatory therapy) within 6 months prior to data collection were excluded.

To study the association between the target polymorphisms and sex, patients were further stratified by sex and each group was compared with their corresponding controls. To analyze the association between the genotypic and allelic distributions of the studied genes and demographic or clinical features of MS, patients were stratified according to age of onset. The age of onset level was set at 30 years as MS primarily manifests in young adults aged 20-40 years (24) and this age is similar to the mean age of onset in the present study. Patients were also divided into two groups based on the EDSS values; the threshold of severe MS was set as an EDSS score of 6 in accordance with a previous study (15). Finally, patients with MS were divided according to the subtype, RRMS and SPMS (25).

The control group was comprised of 152 unrelated healthy subjects (124 females, 28 males; mean age \pm standard deviation, $31.43 \pm 8.31$ years), matched for age and sex with no familial history of MS or any other CNS diseases. These patients were recruited from blood donors in the same geographical area.

In the present study, different genetic models were used to confirm the association between the genes of interest and the risk of MS: i) Genotypic model; in which a wild-type homozygous genotype was used as a reference (AA in both miR-155 rs767649 \& miR-146a rs57095329) to investigate the prevalence of two other genotypes (heterozygous genotypes, AT in miR-155 rs767649 and AG in miR-146a rs57095329, and mutant homozygous genotypes, TT in miR-155 rs767649 and GG in miR-146a rs57095329) in the MS and control groups. ii) Dominant model; in which a wild-type homozygous genotype was used as a reference (AA in both miR-155 rs767649 \& miR-146a rs57095329) to investigate the prevalence of mutant allele associated genotypes (TT+AT in miR-155 rs767649 and GG+AG in miR-146a rs57095329) within the MS and control groups. iii) Recessive model; in which wild-type allele associated genotypes (AA+AT in miR-155 rs767649 and AA+AG in miR-146a rs57095329) was used as a reference to investigate the prevalence of mutant homozygous genotype (TT in miR-155 rs767649 and GG in miR-146a rs57095329) within the MS and control groups. iv) Allelic model; in which a wild-type allele (A allele in both miR-155 rs767649 \& miR-146a rs57095329) was used as a reference to investigate the prevalence of mutant allele ( $\mathrm{T}$ in miR-155 rs767649 and G in miR-146a rs57095329) within the MS and control groups.

All participants provided informed written consent after the study objectives and procedures were explained to them. The present study was approved by The Faculty of Medicine Fayoum University Ethics Committee. The research protocol adhered to the ethical principles and guidelines of The Declaration of Helsinki (26). Peripheral blood samples were collected from all participants and DNA extraction and genotyping were performed.

DNA extraction and genotyping. Genomic DNA was extracted from whole EDTA blood samples from all participants using a QIA-Amplification Extraction kit (Qiagen, Inc.) according to the manufacturer's protocols. DNA concentration was measured using a NanoDrop-1000 Spectrophotometer (NanoDrop Technologies; Thermo Fisher Scientific, Inc.).

All individuals were genotyped for the two SNPs (miR-155 rs767649 $\mathrm{A}>\mathrm{T}$ and miR-146a rs57095329 $\mathrm{A}>\mathrm{G}$ ) by quantitative (q)PCR using a TaqMan Allelic Discrimination assay (Applied Biosystems; Thermo Fisher Scientific, Inc.), using pre-designed primer/probe sets for miR-155 rs767649 A>T (C_2212229_10) and miR-146a rs57095329 A>G (C_90078480_10) (Thermo Fisher Scientific, Inc.). The sequences of the primers used were: miR-155 rs767649 forward, 5'-ATATAACACATT ATCAAAAACACTG-3' and reverse, 5'-CACTTTTCTGAG TGCTCTAATCAGG-3'; and miR-146a rs57095329 forward 5'-CCCCGCGGGGCTGCGGAGAGTACAG-3' and reverse, 5'-CAGGAAGCCTGGGGACCCAGCGCCT-3'.

The thermocycling conditions were as follows: 45 PCR cycles at $92^{\circ} \mathrm{C}$ for $15 \mathrm{sec}$; denaturation at $95^{\circ} \mathrm{C}$ for $10 \mathrm{~min}$; and annealing and extension at $60^{\circ} \mathrm{C}$ for $90 \mathrm{sec}$. Real-time PCR was performed using a Rotor-gene Q Real-Time PCR system (Qiagen, Inc.) (27).

Statistical analysis. Data were analyzed using SPSS version 25 (IBM, Corp.). Data are presented as the mean \pm standard deviation for quantitative variables, as frequencies for the number of cases and as percentages for categorical variables. Comparisons between groups were performed using an ANOVA with a post-hoc Tukey's test for multiple comparisons 
Table I. Comparison of characteristics between patients with MS and controls.

\begin{tabular}{|c|c|c|c|c|}
\hline Parameters & RRMS, n=84 & SPMS, $n=30$ & Control group, $\mathrm{n}=152$ & P-value \\
\hline Age, years, mean \pm SD & \multicolumn{2}{|c|}{$31.32 \pm 8.36$} & $31.43 \pm 8.31$ & 0.928 \\
\hline Age of onset, years, mean \pm SD & $31.25 \pm 7.65$ & $31.50 \pm 10.25$ & - & 0.417 \\
\hline \multirow[t]{2}{*}{ Female/Male, $\%$} & \multicolumn{2}{|c|}{$84.2 / 15.8$} & $81.6 / 18.4$ & 0.365 \\
\hline & $85.7 / 14.3$ & $80 / 20$ & - & 0.560 \\
\hline EDSS, mean \pm SD & $2.46 \pm 1.32$ & $5.75 \pm 1.19$ & - & $<0.001$ \\
\hline
\end{tabular}

MS, multiple sclerosis; RRMS, relapsing-remitting multiple sclerosis; SPMS, secondary progressive multiple sclerosis; EDSS, expanded disability status scale; SD, standard deviation.

or an unpaired t-test in normally distributed quantitative variables; whereas a non-parametric Kruskal-Wallis test with a post-hoc Dunn's test or a Mann-Whitney U test were used for non-normally distributed quantitative variables (28). For comparing categorical information, a $\chi^{2}$ test was used. The Fisher's Exact test was performed as a substitute of the $\chi^{2}$ test when the expected frequency was $>5$ (29). Genotypes and alleles frequencies were analyzed and compared between the MS and the control groups. Univariate and multivariate regression analyses were performed to calculate the odds ratio (OR) with 95\% confidence interval (CI) and adjusted for age and sex as possible confounders. Regression analysis was also performed to determine independent predictors of MS (30). $\mathrm{P}<0.05$ was considered to indicate a statistically significant difference.

Sample size. The sample size was calculated using $\mathrm{G}$ power version 3 (31). The minimal sample size of patients was 100 in each group required to get a power level $0.80, \alpha$ level 0.05 and $9 \%$ as an expected difference in the proportion of subjects with GG in polymorphism miR-146a rs57095329 (A>G) between cases and controls. To overcome the problem of missing data, the calculated sample size was increased by $10 \%$ to reach 110 in each group.

\section{Results}

Demographic and clinical features of patients and controls. The present study included a total of 266 subjects classified into two groups. Group I included 114 patients with MS, 96 females $(84.2 \%)$ and 18 males (15.8\%). A total of $114 \mathrm{MS}$ patients, 84 patients $(73.7 \%)$ were diagnosed with RRMS, whereas 30 patients $(26.3 \%)$ were diagnosed with SPMS. Group II included 152 healthy individuals free from MS or other CNS diseases, including 124 females $(81.6 \%)$ and 28 males $(18.4 \%)$ aged $31.43 \pm 8.31$ years. There was a significant increase in the mean value of EDSS score in SPMS (5.75 \pm 1.19$)$ compared with RRMS $(2.46 \pm 1.32)$ patients $(\mathrm{P}<0.001$; Table I).

Differences in the prevalence of miR-155 rs767649 and miR-146a rs57095329 genotypes and alleles between patients with MS and the controls. The genotypic and allelic distribution of miR-155 rs767649 and miR-146a rs57095329 in patients with MS and controls are presented in Table II. For miR-155 polymorphism in the genotypic and dominant models the AA genotype was used as a reference, the homozygous genotype TT in genotypic and TT+AT in dominant models were associated with significant increases in the risk of MS (adjusted OR, 8.6; 95\% CI, 3.556-20.8; $\mathrm{P}<0.001$ vs. adjusted OR, 3.282; 95\% CI, 1.599-6.738; $\mathrm{P}=0.001$, respectively). Also, in the recessive and allelic models, when the AA+AT genotypes or A allele were used as the reference group, TT genotype in recessive and $\mathrm{T}$ allele in allelic models also showed a significant difference, with higher distribution among patients with MS compared with the controls (both $\mathrm{P}<0.001$ ). However, the prevalence of the heterozygous genotype AT, was significantly higher in controls (63.2\%) compared with patients with MS (55.2\%) (adjusted OR, 2.317; 95\% CI, 1.104-4.864; $\mathrm{P}=0.026$ ).

With regard to the miR-146a polymorphism, using the AA genotype or A allele as a reference, GG and AG genotypes and $\mathrm{G}$ allele were significantly more common in the control groups ( $10.5 \%$ for $\mathrm{GG}, 47.4 \%$ for $\mathrm{AG}$ and $34.2 \%$ for $\mathrm{G}$ allele) compared with patients with MS (2.6\% for GG, 26.3\% for AG and $15.8 \%$ for $\mathrm{G}$ allele). For GG vs. AA the P-value was 0.004 , whereas AG vs. AA and A vs. G allele were both $\mathrm{P}<0.001$. The dominant model (GG+AG vs. AA; adjusted $\mathrm{OR}=0.291$; 95\% CI, 0.173-0.492; $\mathrm{P}<0.001$ ), recessive model (GG vs. $\mathrm{AG}+\mathrm{AA}$; adjusted $\mathrm{OR}=0.233 ; 95 \% \mathrm{CI}, 0.066-0.827 ; \mathrm{P}=0.024)$ were further examined. These data demonstrate that the presence of $\mathrm{G}$ allele was associated with protection for MS, but A allele was associated with the development of the disease.

Stratification of genotype and allele frequencies of miR-155 and miR-146a polymorphisms in MS and control groups according to sex. The miR-155 rs767649 and miR-146a rs57095329 genotypes and alleles were further stratified by sex as shown in Table III. miR-155 rs767649 was significantly associated with an increased risk of MS in females and this was associated with the homozygous TT genotype in the genotypic and recessive models, with the TT+AT genotypes in the dominant model and with $\mathrm{T}$ allele in allelic model (each $\mathrm{P}<0.001$ ). However, the AT genotype was determined to be protective against the development of MS. For miR-146a rs57095329, the $\mathrm{AG}$ in the genotypic model $(\mathrm{P}=0.015), \mathrm{GG}+\mathrm{AG}$ genotypes in the dominant model $(\mathrm{P}=0.001)$ and $\mathrm{G}$ allele in the allelic model $(\mathrm{P}<0.001)$ were significantly associated with a decreased risk of $\mathrm{MS}$.

In the male group, a significant decrease in the risk of MS was observed with miR-146a rs57095329 GG+GA genotypes in the dominant model (adjusted OR=0.053; 95\% CI, 
Table II. Genotype and allele distributions of miR-155 rs767649 A>T and miR-146a rs57095329 A>G in MS cases and control group.

A, $\operatorname{miR}-155 \operatorname{rs} 767649(\mathrm{~A}>\mathrm{T})$

\begin{tabular}{|c|c|c|c|c|}
\hline $\begin{array}{l}\text { Genotypes } \\
\text { and Alleles }\end{array}$ & $\begin{array}{l}\text { MS cases, } \\
\text { n }(\%)\end{array}$ & $\begin{array}{l}\text { Controls, } \\
\mathrm{n}(\%)\end{array}$ & $\begin{array}{l}\text { Unadjusted P-value, } \\
\text { OR (95\% CI) }\end{array}$ & $\begin{array}{c}\text { Adjusted P-value, } \\
\text { OR }(95 \% \text { CI })^{\mathrm{a}}\end{array}$ \\
\hline TT & $39(34.2)$ & $16(10.5)$ & $<0.001,8.125(3.408-19.370)$ & $<0.001,8.600(3.556-20.800)$ \\
\hline AT & $63(55.3)$ & $96(63.2)$ & $0.033,2.187(1.066-4.490)$ & $0.026,2.317$ (1.104-4.864) \\
\hline AA & $12(10.5)$ & $40(26.3)$ & 1 & \\
\hline \multicolumn{5}{|c|}{ Dominant model } \\
\hline $\mathrm{TT}+\mathrm{AT}$ & $102(89.5)$ & $112(73.7)$ & $0.002,3.036(1.510-6.105)$ & $0.001,3.282(1.599-6.738)$ \\
\hline AA & $12(10.5)$ & $40(26.3)$ & 1 & \\
\hline \multicolumn{5}{|c|}{ Recessive model } \\
\hline $\mathrm{TT}$ & $39(34.2)$ & $16(10.5)$ & $<0.001,4.420(2.315-8.438)$ & $<0.001,4.479(2.341-8.571)$ \\
\hline $\mathrm{AA}+\mathrm{AT}$ & $75(65.8)$ & $136(89.5)$ & 1 & \\
\hline Allele T & $141(61.8)$ & $128(42.1)$ & $<0.001,2.228(1.569-3.166)$ & $<0.001,2.260(1.588-3.218)$ \\
\hline Allele A & $87(38.2)$ & $176(57.9)$ & 1 & \\
\hline
\end{tabular}

B, miR-146a rs57095329 (A>G)

\begin{tabular}{lcccc}
\hline $\begin{array}{l}\text { Genotypes } \\
\text { and Alleles }\end{array}$ & $\begin{array}{c}\text { MS cases, } \\
\mathrm{n}(\%)\end{array}$ & $\begin{array}{c}\text { Controls, } \\
\mathrm{n}(\%)\end{array}$ & $\begin{array}{c}\text { Unadjusted P-value, } \\
\text { OR (95\% CI) }\end{array}$ & $\begin{array}{c}\text { Adjusted P-value, } \\
\text { OR (95 \% CI) }\end{array}$ \\
\hline GG & $3(2.6)$ & $16(10.5)$ & $0.003,0.148(0.041-0.531)$ & $0.004,0.149(0.041-0.539)$ \\
AG & $30(26.3)$ & $72(47.4)$ & $<0.001,0.329(0.192-0.564)$ & $<0.001,0.323(0.187-0.556)$ \\
AA & $81(71.1)$ & $64(42.1)$ & & 1 \\
Dominant model & $33(28.9)$ & $88(57.9)$ & $<0.001,0.296(0.177-0.497)$ & $<0.001,0.291(0.173-0.492)$ \\
GG+AG & $81(71.1)$ & $64(42.1)$ & & 1 \\
AA & $3(2.6)$ & $16(10.5)$ & $0.022,0.230(0.065-0.809)$ & $0.024,0.233(0.066-0.827)$ \\
Recessive model & $111(97.4)$ & $136(89.5)$ & & 1 \\
GG & $36(15.8)$ & $104(34.2)$ & $<0.001,0.361(0.235-0.553)$ & $<0.001,0.361(0.235-0.555)$ \\
AA+AG & $192(84.2)$ & $200(65.8)$ & & 1 \\
Allele G & & & \\
Allele A & & & & \\
\hline
\end{tabular}

aAdjusted by age and sex. CI, confidence interval; OR, odds ratio; MS, multiple sclerosis; miR, microRNA.

0.011-0.251; $\mathrm{P}<0.001)$ and with $\mathrm{G}$ allele in the allelic model (adjusted OR=0.225; 95\% CI, 0.080-0.629; $\mathrm{P}=0.004$ ).

Logistical regression analysis. To determine the predictive parameters of increased risk of MS, univariate and multivariate logistical regression analyses were performed (Table IV). For miR-155 rs767649, the TT and AT genotypes in univariate analysis and the TT genotype in the multivariate analysis were positively associated with the development of MS. For miR-146a rs57095329, GG and AG genotypes were inversely associated with MS development in both univariate and multivariate analyses.

Association between miR-155 rs767649 and miR-146a rs57095329 and clinicopathological features of MS. The distribution of miR-155 rs767649 A>T and miR-146a rs57095329 A>G genotypes or alleles among patients with $\mathrm{MS}$ after categorizing them into subgroups according to the age of onset, EDSS and clinical subtypes of the disease are shown in Table V. For miR-155 rs767649, the AT or AT+TT genotypes were significantly associated with increased MS susceptibility among older patients ( $>30$ years) $(\mathrm{P}=0.013)$, higher EDSS score $(\geq 6)(P=0.047)$ and with the SPMS subtype $(P=0.034)$. In contrast, for miR-146a rs57095329, the presence of a $G$ allele or the GG genotype was significantly more frequently observed in patients with a younger age of onset ( $\leq 30$ years) $(P=0.029)$, in patients with a higher EDSS $(\geq 6)(P=0.001)$ and with the SPMS subtype $(\mathrm{P}=0.011)$.

\section{Discussion}

MS is a neuroinflammatory autoimmune disease (1). Variations in the expression of several miRNAs contribute to MS (32). miR-155 and miR-146a expression is frequently increased 
Table III. Stratification of miR-155 and miR-146a polymorphisms by sex in patients with multiple sclerosis and their corresponding healthy controls.

A, miR-155 rs767649 (A>T)

\begin{tabular}{|c|c|c|c|c|c|c|}
\hline Parameters & $\begin{array}{c}\text { Females } \\
\text { cases/controls, \% }\end{array}$ & $\begin{array}{l}\text { Adjusted OR }{ }^{\mathrm{a}} \text {, } \\
\quad(95 \% \mathrm{CI})\end{array}$ & P-value ${ }^{a}$ & $\begin{array}{c}\text { Males } \\
\text { cases/controls, \% }\end{array}$ & $\begin{array}{l}\text { Adjusted } \mathrm{OR}^{\mathrm{a}} \text {, } \\
\quad(95 \% \mathrm{CI})\end{array}$ & P-value \\
\hline TT & $37.5 / 6.5$ & $19.112,(6.495-56.233)$ & $<0.001$ & $16.7 / 28.6$ & $0.488,(0.065-3.682)$ & 0.486 \\
\hline AT & $53.1 / 64.5$ & $2.773,(1.180-6.516)$ & 0.019 & $66.7 / 57.1$ & $0.975,(0.179-5.306)$ & 0.976 \\
\hline AA & $9.4 / 29$ & 1 & & $16.7 / 14.3$ & 1 & \\
\hline \multicolumn{7}{|c|}{ Dominant model } \\
\hline $\mathrm{TT}+\mathrm{AT}$ & $90.6 / 71$ & $4.540,(1.983-10.396)$ & $<0.001$ & $83.3 / 85.7$ & $0.813,(0.156-4.229)$ & 0.806 \\
\hline AA & $9.4 / 29$ & 1 & & $16.7 / 14.3$ & 1 & \\
\hline \multicolumn{7}{|c|}{ Recessive model } \\
\hline $\mathrm{TT}$ & $37.5 / 6.5$ & $8.717,(3.811-19.943)$ & $<0.001$ & $16.7 / 28.6$ & $0.498,(0.112-2.207)$ & 0.359 \\
\hline $\mathrm{AA}+\mathrm{AT}$ & $62.5 / 93.5$ & 1 & & $83.3 / 71.4$ & 1 & \\
\hline Allele $\mathrm{T}$ & $64.1 / 38.7$ & $2.863,(1.932-4.243)$ & $<0.001$ & $50 / 57.1$ & $0.744,(0.320-1.730)$ & 0.493 \\
\hline Allele A & $35.9 / 61.3$ & 1 & & $50 / 42.9$ & 1 & \\
\hline
\end{tabular}

B, miR-146a rs57095329 (A>G)

\begin{tabular}{|c|c|c|c|c|c|c|}
\hline Parameters & $\begin{array}{c}\text { Females } \\
\text { cases/controls, \% }\end{array}$ & $\begin{array}{l}\text { Adjusted OR }{ }^{\mathrm{a}} \text {, } \\
\quad(95 \% \mathrm{CI})\end{array}$ & P-value ${ }^{a}$ & $\begin{array}{c}\text { Males } \\
\text { cases/controls, \% }\end{array}$ & $\begin{array}{l}\text { Adjusted OR }{ }^{\mathrm{a}} \text {, } \\
\quad(95 \% \mathrm{CI})\end{array}$ & P-value ${ }^{a}$ \\
\hline GG & $0 / 9.7$ & - & 0.999 & $16.7 / 14.3$ & $0.316,(0.051-1.979)$ & 0.218 \\
\hline $\mathrm{AG}$ & $31.2 / 43.5$ & $0.487,(0.273-0.869)$ & 0.015 & $0 / 64.3$ & - & 0.999 \\
\hline AA & $68.8 / 46.8$ & 1 & & $83.3 / 21.4$ & 1 & \\
\hline \multicolumn{7}{|c|}{ Dominant model } \\
\hline $\mathrm{GG}+\mathrm{AG}$ & $31.2 / 53.2$ & $0.392,(0.223-0.689)$ & 0.001 & $16.7 / 78.6$ & $0.053,(0.011-0.251)$ & $<0.001$ \\
\hline $\mathrm{AA}$ & $68.8 / 46.8$ & 1 & & $83.3 / 21.4$ & 1 & \\
\hline \multicolumn{7}{|c|}{ Recessive model } \\
\hline GG & $0 / 9.7$ & - & 0.999 & $16.7 / 14.3$ & $1.175,(0.223-6.193)$ & 0.849 \\
\hline $\mathrm{AA}+\mathrm{AG}$ & $100 / 90.3$ & 1 & & $83.3 / 85.7$ & 1 & \\
\hline Allele G & $15.6 / 31.5$ & $0.403,(0.251-0.647)$ & $<0.001$ & $16.7 / 46.4$ & $0.225,(0.080-0.629)$ & 0.004 \\
\hline Allele A & $84.4 / 68.5$ & 1 & & $83.3 / 53.6$ & 1 & \\
\hline
\end{tabular}

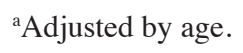

in MS peripheral blood mononuclear cells $(7,17)$. Therefore, these two miRNAs may be used as a signature for determining susceptibility to MS and serve crucial roles in the pathogenesis of MS. Previous studies have investigated the association between miR-155 rs767649 and miR-146a rs57095329 polymorphisms with MS susceptibility reporting no difference in the frequencies of miR-155 rs767649 and miR-146a rs57095329 between patients with MS and controls $(17,21)$. Therefore, the present study aimed to investigate the association of these two polymorphisms with MS susceptibility and their association with clinicopathological data in the Egyptian population specifically.

In the present case-control study, it was demonstrated for the first time that miR-155 rs767649 (A>T) was a predisposing factor for MS in all genetic and allelic models. Univariate and multivariate logistical regression analyses demonstrated that the TT genotype was a risk factor for MS susceptibility. In contrast to the results of the present study, a recent study showed that there was no association between miR-155 rs767649 and MS risk in Iranian patients (21). Additionally, in a previous study examining the association between miR-155 rs767649 and susceptibility to type 1 diabetes mellitus (another autoimmune disease), miR-155 rs767649 was associated with protection against this disease (16).

The results of the present study may be explained by previous studies which demonstrated that the miR-155 rs767649 TT genotype and T allele were associated with increased risk of non-small cell lung cancer (NSCLC) and hepatocellular carcinoma ( $\mathrm{HCC})$, respectively, wherein the miR-155 rs767649 $\mathrm{T}$ allele contributed to higher expression 
Table IV. Logistic regression analysis to predict the risk of Multiple Sclerosis.

A, Univariate analysis

\begin{tabular}{lccrrr}
\hline Parameter & Unstandardized coefficient & Standard error for estimate & P-value & Odds ratio & 95\% confidence interval \\
\hline miR-155 (TT) & 2.095 & 0.443 & $<0.001$ & 8.125 & $3.408-19.370$ \\
miR-155 (AT) & 0.783 & 0.367 & 0.033 & 2.187 & $1.066-4.490$ \\
miR-146a (GG) & $-1.910-$ & 0.651 & 0.003 & 0.148 & $0.0421-0.531$ \\
miR-146a (AG) & $-1.111-$ & 0.274 & $<0.001$ & 0.329 & $0.192-0.564$ \\
\hline
\end{tabular}

B, Multivariate analysis

\begin{tabular}{lccrcc}
\hline Parameter & Unstandardized coefficient & Standard error for estimate & P-value & Odds ratio & 95\% confidence interval \\
\hline miR-155 (TT) & 2.504 & 0.496 & $<0.001$ & 12.236 & $4.624-32.376$ \\
miR-155 (AT) & 0.519 & 0.391 & 0.184 & 1.681 & $0.782-3.615$ \\
miR-146a (GG) & $-2.893-$ & 0.739 & $<0.001$ & 0.055 & $0.013-0.236$ \\
miR-146a (AG) & $-1.341-$ & 0.319 & $<0.001$ & 0.262 & $0.140-0.489$ \\
\hline
\end{tabular}

miR, microRNA.

Table V. Association between single nucleotide polymorphisms of miR-155 rs767649 and miR-146a rs57095329 and clinical data of patients with multiple sclerosis.

\begin{tabular}{|c|c|c|c|c|c|c|c|c|c|}
\hline \multirow[b]{2}{*}{ Genotypes/alleles } & \multicolumn{2}{|c|}{ Age of onset, years } & \multirow[b]{2}{*}{ P-value } & \multicolumn{2}{|c|}{ EDSS } & \multirow[b]{2}{*}{ P-value } & \multicolumn{2}{|c|}{ MS type } & \multirow[b]{2}{*}{ P-value } \\
\hline & $\begin{array}{c}>30, \mathrm{n}=57 \\
\mathrm{n}(\%)\end{array}$ & $\begin{array}{c}\leq 30, \mathrm{n}=57 \\
\mathrm{n}(\%)\end{array}$ & & $\begin{array}{c}\geq 6, n=24 \\
n(\%)\end{array}$ & $\begin{array}{c}<6, \mathrm{n}=90 \\
\mathrm{n}(\%)\end{array}$ & & $\begin{array}{c}\text { RRMS, n=84 } \\
\text { n (\%) }\end{array}$ & $\begin{array}{c}\text { SPMS, } n=30 \\
n(\%)\end{array}$ & \\
\hline miR-155 (A/T) & & & $0.013^{\mathrm{a}}$ & & & $0.047^{\mathrm{a}}$ & & & 0.088 \\
\hline $\mathrm{AA}$ & $3(5.3)$ & $9(15.8)$ & & 0 & $12(13.3)$ & & $12(14.3)$ & $0(0.0)$ & \\
\hline $\mathrm{AT}$ & $39(68.4)$ & $24(42.1)$ & & $18(75.0)$ & $45(50.0)$ & & $45(53.6)$ & $18(60.0)$ & \\
\hline TT & $15(26.3)$ & $24(42.1)$ & & $6(25.0)$ & $33(36.7)$ & & $27(32.1)$ & $12(40.0)$ & \\
\hline $\mathrm{AT}+\mathrm{TT}$ & $54(94.7)$ & $48(84.2)$ & 0.067 & $24(100.0)$ & $78(86.7)$ & 0.068 & $72(85.7)$ & $30(100.0)$ & 0.034 \\
\hline Allele & & & 0.683 & & & 0.916 & & & 0.131 \\
\hline A & $45(39.5)$ & $42(36.8)$ & & $69(41.1)$ & $18(30.0)$ & & $18(37.5)$ & $69(38.3)$ & \\
\hline $\mathrm{T}$ & $69(60.5)$ & $72(63.2)$ & & $99(58.9)$ & $42(70.0)$ & & $30(62.5)$ & $111(61.7)$ & \\
\hline miR-146a (A/G) & & & 0.074 & & & $0.001^{\mathrm{b}}$ & & & $0.011^{\mathrm{b}}$ \\
\hline AA & $45(78.9)$ & $36(63.2)$ & & $18(75.0)$ & $63(70.0)$ & & $60(71.4)$ & $21(70.0)$ & \\
\hline $\mathrm{AG}$ & $12(21.1)$ & $18(31.6)$ & & $3(12.5)$ & $27(30.0)$ & & $24(28.6)$ & $6(20.0)$ & \\
\hline GG & 0 & $3(5.3)$ & & $3(12.5)$ & 0 & & 0 & $3(10.0)$ & \\
\hline $\mathrm{AG}+\mathrm{GG}$ & $12(21.1)$ & $21(36.8)$ & 0.063 & $6(25.0)$ & $27(30.0)$ & 0.631 & $24(28.6)$ & $9(30.0)$ & 0.882 \\
\hline Allele & & & 0.029 & & & 0.527 & & & 0.297 \\
\hline A & $102(89.5)$ & $90(78.9)$ & & $144(85.7)$ & $48(80.0)$ & & $39(81.3)$ & $153(85.0)$ & \\
\hline $\mathrm{G}$ & $12(10.5)$ & $24(21.1)$ & & $24(14.3)$ & $12(20.0)$ & & $9(18.8)$ & $27(15.0)$ & \\
\hline
\end{tabular}

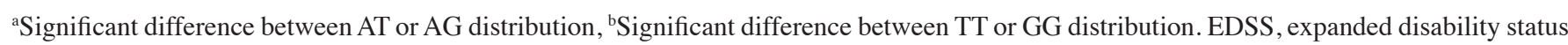
scale; MS, multiple sclerosis; RRMS, relapsing-remitting multiple sclerosis; SPMS, secondary progressive multiple sclerosis.

level of miR-155 in NSCLC and HCC tissues and in adjacent non-tumor tissues, potentially via enhanced transcriptional activity $(18,19)$. In addition, miR-155 expression level was upregulated in patients with MS (7) and in EAE in mice (10).
In MS and EAE, miR-155 induced the development of Th1 and Th17 cells with a subsequent increase in the release of proinflammatory cytokines (33). Therefore, targeted inhibition of miR-155 activity may be a novel therapeutic approach for 
MS $(7,33)$. The results of these former studies informed the hypothesis of the present study that the miR-155 rs767649 polymorphism may be associated with increased risk of MS.

Regarding the miR-146a rs57095329 (A>G) polymorphism, the present study demonstrated for the first time that the mutated $\mathrm{G}$ allele confers protection against MS development in all genetic models. In addition, both univariate and multivariate logistic regression analyses showed that the GG genotype is negatively associated with MS susceptibility. However, these data concerning miR-146a contradict a previous study investigating MS in the Chinese population, which concluded that the miR-146a rs57095329 (A>G) polymorphism was not associated with MS (17). In addition, the present study contradicts the results of a study on another autoimmune disease which showed that an increased risk of systemic lupus erythematosus (SLE) in Chinese population was associated with the miR-146a rs57095329 mutant allele (20). These disparities may be due to ethnic and possibly lifestyle differences in the studied populations, or due to the different nature of the studied disease in the case of SLE.

It was reported that the protein-binding affinity of the transcription factor Ets-1 was decreased by the G allele of miR-146a rs57095329, leading to reduced miR-146a expression level in patients with SLE (20). Also, it was found that the G allele of miR-146a rs57095329 was linked to the diminished expression of miR-146a in patients with RRMS (17), patients with severe sepsis (34) even in healthy controls (20). Previous studies have reported that miR-146a expression level was increased in MS and EAE lesions of mice and that this was associated with increased production of the MS related proinflammatory cytokine IL-17 by inhibiting the target gene IRAK1 $(35,36)$. These previous studies, which concluded that increased miR-146a was associated with MS risk and the mutant $G$ allele was associated with decreased expression level, support the results of the present study showing that the miR-146a rs57095329 $(A>G)$ polymorphism decreased miR-146a expression level and was associated with a decreased risk of MS.

Previous studies have reported that MS is more prevalent in females compared with males (37), therefore the present study evaluated the effect of miR-155 rs767649 and miR-146a rs57095329 on MS risk in both sexes. Notably, the miR-155 rs767649 A>T polymorphism was associated with risk of MS in females, but not in males; however, miR-146a rs57095329 A $>$ G functioned as a defense polymorphism in both sexes. A previous study demonstrated that the estrogen induced immunomodulatory effects in MS ranged from anti-inflammatory to pro-inflammatory effects, based on the woman's reproductive age (38), therefore the sex-dependent relationship observed in the present study between polymorphisms of miRNAs and MS susceptibility may be due to differences in endocrine profiles between males and females. It was previously shown that estrogen treatment increased miR-155 expression level (39) and decreased the expression level of miR-146a (40). Moreover, serum miR-146a concentrations were lower in healthy postmenopausal women who used hormonal replacement therapy compared with their control non-using monozygotic twins (41). Therefore, females with miR-155 rs767649 in the present study may be more susceptible to the development of MS compared with males possessing similar polymorphism; however, females with
miR-146a rs57095329 are less likely to develop MS compared with males carrying the same polymorphism.

The present study revealed that higher EDSS values were significantly associated with SPMS compared with RRMS. A recent study reported that a high EDSS value is one of the defining criteria for the diagnosis of SPMS (42). SPMS is the secondary stage of the disease developing within 15-20 years after the initial RRMS diagnosis, and is characterized by progressive deterioration of neurological functions with accumulating disability over time (25). This may explain the significant association between higher EDSS values and the SPMS subgroup compared with patients with RRMS.

Regarding miR-155 rs767649, the AT/TT genotypes were significantly associated with higher EDSS values and the SPMS subtype. These findings were consistent with previous reports showing that the miR-155 rs767649 $\mathrm{T}$ allele increases expression level of miR-155 $(18,19)$ subsequently enhancing miR-155 activity (43) and leading to worsened disability and neurological dysfunction (44), and this may underlie the association between SPMS and higher EDSS values $(23,25)$.

There was a significantly higher prevalence of miR-155 rs767649 AT in the controls, and this was significantly associated with an older age of onset. Additionally, as miR-146a rs57095329 A>G was found to be protective in the present study, it was hypothesized that it may be associated with an older age of onset, lower EDSS scores and a less severe MS subtype (RRMS); however, the reverse was observed. These unexpected results may be due to a combination of differences in genetic susceptibility and environmental factors, both of which are implicated in MS causation. For example, previous studies have reported that environmental factors such as deficiency of vitamin D, Epstein Barr Virus infection and smoking may increase the risk of MS in genetically predisposed patients $(3,4,5)$ as well as influence the expression levels of miR-155 and miR-146a (45-47) and consequently impacting the activity, disability and subtype of patients $(43,44)$. Therefore, other functional polymorphisms of miR-155 and miR-146a genes should also be investigated to determine their association with susceptibility to MS.

In summary, both SNPs of the miR-155 \& miR-146a investigated in the present study were associated with susceptibility to MS and this knowledge may aid our understanding of the molecular mechanisms underlying the pathogenesis and progression of MS and in the diagnosis and development of improved therapies for treatment of MS. The present study identifies potentially novel diagnostic biomarkers for MS susceptibility in the Egyptian population. Additionally, as miR-155 rs767649 was associated with an increased female-specific MS risk in the present study, this may be used as an additional biomarker for diagnosis. Previous studies have demonstrated that identifying MS risk or protective genes may have important implications on the parental transmission of MS (48). However, replication of the results of the present study in a larger cohort of patients with MS and healthy controls is required.

To the best of our knowledge, the present study is the first to report a positive association between miR-155 rs767649 and miR-146a rs57095329 polymorphisms with susceptibility to MS, particularly in females. Furthermore, the present study is the first to investigate the contribution of these two SNPs 
to MS susceptibility in an Egyptian cohort. These results may improve our understanding of the clinical significance of the studied polymorphisms in the susceptibility to MS.

The present study had some limitation. First, due to the hospital-based and case-control nature of the study, selection bias was unavoidable. Second, there was a lack of data concerning other genetic and environmental risk factors contributing to MS development for these patients, therefore gene-gene and gene-environment interactions could not be assessed. Third, due to the small number of patients involved, the results of the present study need to be confirmed in future studies with larger cohorts, and the involvement of other functional polymorphisms in the studied genes should also be assessed.

In conclusion, miR-155 rs767649 and miR-146a rs57095329 SNPs were associated with the risk of developing MS in an Egyptian cohort, particularly in females. miR-155 rs767649 was associated with an increased risk of MS and miR-146a rs57095329 was associated with a decreased risk. These novel findings may be clinically significant and highlight the role of miR-155 and miR-146a in the pathogenesis of MS, with possible therapeutic implications.

\section{Acknowledgements}

The authors would like to thank the help from Dr Muhammad Masoud (Fayoum University, Egypt).

\section{Funding}

No funding was received.

\section{Availability of data and materials}

The datasets analyzed in the present study are not publicly available due to the confidentiality of patients' information.

\section{Authors' contributions}

MAA designed the study. OGS, EEM, SNG, HME and EME gathered the patient data and collected patient blood samples. MAA, OGS performed the experiments and analyzed the data. MAA, EME, SNG, HME and EEM wrote the manuscript. All authors read and approved the final manuscript.

\section{Ethics approval and consent to participate}

The present study was approved by The Faculty of Medicine, Fayoum University Ethics Committee and all participants provided informed written consent. The research protocol complied with the ethical principles and guidelines of the Declaration of Helsinki.

\section{Patients consent for publication}

Not applicable.

\section{Competing interests}

The authors declare that they have no competing interests.

\section{References}

1. Ehya F, Abdul Tehrani H, Garshasbi M and Nabavi SM Identification of miR-24 and miR-137 as novel candidate multiple sclerosis miRNA biomarkers using multi-staged data analysis protocol. Mol Biol Res Commun 3: 127-140, 2017.

2. Lassmann H, van HJ and Mahad D: Progressive multiple sclerosis: Pathology and pathogenesis. Nat Rev Neurol 8: 647-656, 2012.

3. Oksenberg JR and Barcellos LF: Multiple sclerosis genetics: Leavin no stone unturned. Genes Immun 6: 375-387, 2005.

4. Taylor BV: The major cause of multiple sclerosis is environmental: Genetics has a minor role-yes. Mult Scler 17: 1171-1173, 2011.

5. O'Gorman C, Lucas R and Taylor B: Environmental risk factors for multiple sclerosis: A review with a focus on molecular mechanisms. Int J Mol Sci 13: 11718-11752, 2012.

6. $\mathrm{Xu}$ WD, Lu MM, Pan HF and Ye DQ: Association of MicroRNA-146a with autoimmune diseases. Inflammation 35: 1525-1529, 2012.

7. Paraboschi EM, Soldà G, Gemmati D, Orioli E, Zeri G, Benedetti MD, Salviati A, Barizzone N, Leone M, Duga S and Asselta R: Genetic association and altered gene expression of mir-155 in multiple sclerosis patients. Int J Mol Sci 12: 8695-8712, 2011

8. Rothchild AC, Sissons JR, Shafiani S, Plaisier C, Min D, Mai D, Gilchrist M, Peschon J, Larson RP, Bergthaler A, et al: MiR-155-regulated molecular network orchestrates cell fate in the innate and adaptive immune response to Mycobacterium tuberculosis. Proc Natl Acad Sci USA 113: E6172-E6181, 2016.

9. Min M, Peng L, Yang Y, Guo M, Wang W and Sun G: MicroRNA-155 is involved in the pathogenesis of ulcerative colitis by targeting FOXO3a. Inflamm Bowel Dis 20: 652-659, 2014.

10. Murugaiyan G, Beynon V, Mittal A, Joller N and Weiner HL: Silencing microRNA-155 ameliorates experimental autoimmune encephalomyelitis. J Immunol 187: 2213-2221, 2011.

11. Taganov KD, Boldin MP, Chang KJ and Baltimore D: NF- $\kappa \mathrm{B}$-dependent induction of microRNA miR-146, an inhibitor targeted to signaling proteins of innate immune responses. Proc Natl Acad Sci USA 103: 12481-12486, 2006.

12. Leng RX, Pan HF, Qin WZ, Chen GM and Ye DQ: Role of microRNA-155 in autoimmunity. Cytokine Growth Factor Rev 22: 141-147, 2011

13. Lescher J, Paap F, Schultz V, Redenbach L, Scheidt U, Rosewich H, Nessler S, Fuchs E, Gärtner J, Brück W and Junker A: MicroRNA regulation in experimental autoimmune encephalomyelitis in mice and marmosets resembles regulation in human multiple sclerosis lesions. J Neuroimmunol 246: 27-33, 2012.

14. Bell JI: Single nucleotide polymorphisms and disease gene mapping. Arthritis Res 4 (Suppl 3): S273-S278, 2002.

15. Labib DA, Shaker OG, El Refai RM, Ghoniem SA and Elmazny A: Association between miRNA-146a and polymorphisms of its target gene, IRAK1, regarding susceptibility to and clinical features of systemic lupus erythematous and multiple sclerosis. Lab Med 50: 34-41, 2019.

16. Assmann1 TS, Duarte GCK, Brondani LA, de Freitas PHO, Martins ÉM, Canani1 LH and Crispim D: Polymorphisms in genes encoding miR-155 and miR-146a are associated with protection to type 1 diabetes mellitus. Acta Diabetol 54: 433-441, 2017.

17. Li Y, Du C, Wang W, Ma G, Cui L, Zhou H, Tao H, Yao L, Zhao B and Li K: Genetic association of MiR-146a with multiple sclerosis susceptibility in the Chinese population. Cell Physiol Biochem 35: 281-291, 2015.

18. Xie K, Ma H, Liang C, Wang C, Qin N, Shen W, Gu Y, Yan C, Zhang K, Dai N, et al: A functional variant in miR-155 regulation region contributes to lung cancer risk and survival. Oncotarget 6 : 42781-42792, 2015.

19. Ji J, Xu M, Tu J, Zhao Z, Gao J, Chen M, Song J, Zhu H, Cheng X, Hui J, et al: MiR-155 and its functional variant rs767649 contribute to the susceptibility and survival of hepatocellular carcinoma. Oncotarget 7: 60303-60309, 2016.

20. Luo X, Yang W, Ye DQ, Cui H, Zhang Y, Hirankarn N, Qian X, Tang Y, Lau YL, de Vries N, et al: A functional variant in microRNA-146a promoter modulates its expression and confers disease risk for systemic lupus erythematosus. PLoS Genet 7: e1002128, 2011. 
21. Golshani Z, Hojati Z, Sharifzadeh A, Shaygannejad V and Jafarinia M: Genetic variation in intergenic and exonic miRNA sequence and risk of multiple sclerosis in the isfahan patients. Iran J Allergy Asthma Immunol 17: 477-484, 2018.

22. McDonald WI, Compston A, Edan G, Goodkin D, Hartung HP, Lublin FD, McFarland HF, Paty DW, Polman CH, Reingold SC, et al: Recommended diagnostic criteria for multiple sclerosis: Guidelines from the international panel on the diagnosis of multiple sclerosis. Ann Neurol 50: 121-127, 2001.

23. Kurtzke JF: Rating neurologic impairment in multiple sclerosis: An expanded disability status scale (EDSS). Neurology 33: 1444-1452, 1983

24. Lotti CBC, Oliveira ASB, Bichuetti DB, Castro I and Oliveira EML: Late onset multiple sclerosis: Concerns in aging patients. Arq Neuropsiquiatr 75: 451-456, 2017.

25. Hurwitz BJ: The diagnosis of multiple sclerosis and the clinical subtypes. Ann Indian Acad Neurol 12: 226-230, 2009.

26. Puri KS, Suresh KR, Gogtay NJ and Thatte UM: Declaration of Helsinki, 2008: Implications for stakeholders in research. J Postgrad Med 55: 131-134, 2009.

27. Shaker OG, El Boghdady NA and El Sayed AE: Association of MiRNA-146a, MiRNA-499, IRAK1 and PADI4 polymorphisms with rheumatoid arthritis in Egyptian population. Cell Physiol Biochem 46: 2239-2249, 2018

28. Chan YH: Biostatistics 102: Quantitative data-parametric \& non-parametric tests. Singapore Med J 44: 391-396, 2003.

29. Chan YH: Biostatistics 103: Qualitative data-tests of independence. Singapore Med J 44: 498-503, 2003.

30. Chan YH: Biostatistics 202: Logistic regression analysis. Singapore Med J 45: 149-153, 2004.

31. Faul F, Erdfelder E, Lang AG and Buchner A: G*Power 3: A flexible statistical power analysis program for the social, behavioral, and biomedical sciences. Behav Res Methods 39: 175-191, 2007.

32. Kiselev I,Bashinskaya V, Kulakova O,Baulina N,PopovaE,Boyko A and Favorova O: Variants of MicroRNA genes: Gender-specific associations with multiple sclerosis risk and severity. Int J Mol Sci 16: 20067-20081, 2015

33. Zhang J, Cheng Y, Cui W, Li M, Li B and Guo L: MicroRNA-155 modulates Th1 and Th17 cell differentiation and is associated with multiple sclerosis and experimental autoimmune encephalomyelitis. J Neuroimmunol 266: 56-63, 2014.

34. Shao Y, Li J, Cai Y, Xie Y, Ma G, Li Y, Chen Y, Liu G, Zhao B, Cui $\mathrm{L}$ and $\mathrm{Li} \mathrm{K}$ : The functional polymorphisms of miR-146a are associated with susceptibility to severe sepsis in the Chinese population. Mediators Inflamm 2014: 916202, 2014.

35. Ghaffari SA, Nemati M, Hajghani H, Ebrahimi H, Sheikhi A and Jafarzadeh A: Circulating concentrations of interleukin (IL)-17 in patients with multiple sclerosis: Evaluation of the effects of gender, treatment, disease patterns and IL-23 receptor gene polymorphisms. Iran J Neurol 16: 15-25, 2017.
36. Xia P, Fang X, Zhang ZH, Huang Q, Yan KX, Kang KF, Han L and Zheng ZZ: Dysregulation of miRNA146a versus IRAK1 induces IL-17 persistence in the psoriatic skin lesions. Immunol Lett 148: 151-162, 2012

37. Bove R and Chitnis T: Sexual disparities in the incidence and course of MS. Clin Immunol 149: 201-210, 2013.

38. Ysrraelit MC and Correale J: Impact of sex hormones on immune function and multiple sclerosis development. Immunology 156 : 9-22, 2019.

39. Zhang C, Zhao J and Deng H: $17 \beta$-estradiol up-regulates miR-155 expression and reduces TP53INP1 expression in MCF-7 breast cancer cells. Mol Cell Biochem 379: 201-211, 2013.

40. Dai R, Phillips RA, Zhang Y, Khan D, Crasta O and Ahmed SA: Suppression of LPS-induced Interferon-gamma and nitric oxide in splenic lymphocytes by select estrogen-regulated microRNAs: A novel mechanism of immune modulation. Blood 112: 4591-4597, 2008.

41. Kangas R, Pöllänen E, Rippo MR, Lanzarini C, Prattichizzo F, Niskala P, Jylhävä J, Sipilä S, Kaprio J, Procopio AD, et al: Circulating miR-21, miR-146a and Fas ligand respond to postmenopausal estrogen-based hormone replacement therapy-a study with monozygotic twin pairs. Mech Ageing Dev 143-144: 1-8, 2014.

42. Bsteh G, Ehling R, Lutterotti A, Hegen H, Di Pauli F, Auer M, Deisenhammer F, Reindl $\mathrm{M}$ and Berger T: Long term clinical prognostic factors in relapsing-remitting multiple sclerosis: Insights from a 10-year observational study. PLoS One 11: e0158978, 2016.

43. Chen C, Zhou Y, Wang J, Yan Y, Peng L and Qiu W: Dysregulated MicroRNA involvement in multiple sclerosis by induction of T helper 17 cell differentiation. Front Immunol 9: 1256, 2018.

44. Goodin DS, Reder AT, Bermel RA, Cutter GR, Fox RJ, John GR, Lublin FD, Lucchinetti CF, Miller AE, Pelletier D, et al: Relapses in multiple sclerosis: Relationship to disability. Mult Scler Relat Disord 6: 10-20, 2016.

45. Karkeni E, Bonnet L, Marcotorchino J, Tourniaire F, Astier J, Ye J and Landrier JF: Vitamin D limits inflammation-linked microRNA expression in adipocytes in vitro and in vivo: A new mechanism for the regulation of inflammation by vitamin D. Epigenetics 13: 156-162, 2018.

46. Lu F, Weidmer A, Liu CG, Volinia S, Croce CM and Lieberman PM: Epstein-Barr virus-induced miR-155 attenuates NF-kappaB signaling and stabilizes latent virus persistence. J Virol 82: 10436-20443, 2008.

47. Momi N, Kaur S, Rachagani S, Ganti AK and Batra SK: Smoking and microRNA dysregulation: A cancerous combination. Trends Mol Med 20: 36-47, 2014.

48. Harbo HF, Gold R and Tintoré M: Sex and gender issues in multiple sclerosis. Ther Adv Neurol Disord 6: 237-248, 2013. 